\title{
RISCOS ASSOCIADOS AO ANTICONCEPCIONAL DE EMERGÊNCIA
}

\author{
Julison Andre Perreira Ferreira ${ }^{\mathrm{I}}$ \\ Rosiane Arcanjo da Silva ${ }^{2}$ \\ Paulo Sérgio Ferreira de Lima ${ }^{3}$
}

RESUMO: O contraceptivo de emergência (CE), é uma substância hormonal composta por levonorgestrel, um progestágeno isolado, conhecido popularmente como pílula do dia seguinte, bastante utilizado no Brasil para evitar gravidez indesejada, esse medicamento deve ser administrado logo após o coito sexual ou no máximo em até 120 horas após o ato sexual. Embora esse método seja prático, o uso frequente pode levar a perda ou diminuição da eficácia, desencadeando problemas hormonais, e de infertilidade. Este estudo descreve o mecanismo de ação do CE, identicar riscos associados ao uso continuo e os seus efeitos adversos mais comuns. Trata-se de um estudo de revisão de literatura qualitativo descritivo, sobre os riscos associados ao uso de contracepção de emergência, realizados por meios de estudos e informações obtidas com pesquisas em fontes como artigos científicos. Os artigos utilizados para o desenvolvimento desse trabalho foram encontrados em bases de dados como sites, Literatura Latino-americana e do Caribe em Ciências da Saúde (LILACS), Scientific Eletronic Library Online (SCIELO) e o Google Acadêmico. O CE não tem um mecanismo de ação preciso, mas sabe-se que ele altera processos fisiológicos da fecundação. Previne a ocorrência da ovulação, interfere na fertilização, com o transporte do embrião para o útero, ou inibe a implantação no endométrio. De acordo com a Organização Mundial de Saúde (OMS) relata que o CE tem alta concentração hormonal, seu uso em demasia pode prejudicar a fertilização do óvulo, em vista disso ele é contra indicado para mulheres que desejam engravidar futuramente e para grávidas que possuem riscos de doenças ginecológicas causadas por diferentes parceiros e também em paciente com risco de trombose vascular, tromboembolismo, diabéticos com complicação vascular, hipertensão descontrolada, doenças sanguíneas, pessoas obesas, mulheres que tem o fluxo menstrual em excesso. O CE é uma verdadeira bomba de hormônios é pode conter dez vez mais hormônios comparados ao contraceptivo convencional, o uso abusivo pode causar vários danos ao organismo feminino, bem como, efeitos adversos que envolvem além de náuseas e vômitos, efeitos tromboembólicos e outras reações tensão mamarias, hemorragia vaginal, fadiga, cefaleias, vertigens, astenia e dores na pélvica. Conclui-se, CE tem como função impedir uma possível gravidez indesejada após coito desprotegida possuindo assim suas vantagens e desvantagens. Contudo deve ser usado com cautela, já que o mesmo provoca uma sobrecarga hormonal no organismo feminino, gerando serias consequências para sua usuária, isso quando usada em excesso e sem conhecimento prévio, por ser um medicamento de fácil acesso.

Palavras-chave: Contraindicado. Mecanismo de ação. Pílula do dia seguinte. Riscos do uso do CE.

\footnotetext{
I Acadêmico de Farmácia da Universidade da Amazônia -UNAMA.

2Bacharelado em farmácia - Universidade da Amazônia -UNAMA. E-mail: rosianearcanjo@gmail.com.

${ }^{3}$ Docente na universidade da Amazônia (Unama). Mestrado em biociencias (UFOPA). Especialização em

farmacologia clínica e prescrição farmacêutica (FACES). Graduado em Bacharel em Farmácia.
} 
ABSTRACT: Emergency contraceptive (EC) is a hormonal substance composed of levonorgestrel, an isolated progestin, popularly known as the morning-after pill, widely used in Brazil to prevent unwanted pregnancy, this drug should be administered soon after sexual intercourse or up to I2o hours after the sexual act. Although this method is practical, frequent use can lead to loss or decrease in effectiveness, triggering hormonal problems and infertility. This study describes the mechanism of action of EC, identifying risks associated with continued use and its most common adverse effects. This is a descriptive qualitative literature review study on the risks associated with the use of emergency contraception, carried out by means of studies and information obtained from research in sources such as scientific articles. The articles used for the development of this work were found in databases such as websites, Latin American and Caribbean Literature in Health Sciences (LILACS), Scientific Electronic Library Online (SCIELO) and Academic Google. EC does not have a precise mechanism of action, but it is known to alter the physiological processes of fertilization. It prevents the occurrence of ovulation, interferes with fertilization, with the transport of the embryo to the uterus, or inhibits implantation in the endometrium. According to the World Health Organization (WHO) reports that EC has a high hormonal concentration, its use in excess can harm the fertilization of the egg. of gynecological diseases caused by different partners and also in patients at risk of vascular thrombosis, thromboembolism, diabetics with vascular complications, uncontrolled hypertension, blood diseases, obese people, women who have excessive menstrual flow. EC is a true hormone pump and can contain ten times more hormones compared to conventional contraceptives, abuse can cause several damages to the female body, as well as adverse effects that involve, in addition to nausea and vomiting, thromboembolic effects and other tension reactions breasts, vaginal bleeding, fatigue, headache, dizziness, asthenia and pelvic pain. In conclusion, EC has the function of preventing a possible unwanted pregnancy after unprotected intercourse, thus having its advantages and disadvantages. However, it should be used with caution, as it causes a hormonal overload in the female body, generating serious consequences for its user, when used in excess and without prior knowledge, as it is an easily accessible drug.

Keywords: Contraindicated. Mechanism of action. Pill of the next day. Risks of using the CE.

\section{INTRODUÇÃO}

O contraceptivo de emergência (CE), é uma substância hormonal composta por levonorgestrel, um progestágeno isolado, conhecido popularmente como pílula do dia seguinte, bastante utilizado no Brasil para evitar gravidez indesejada seja ela pelo uso incorreto de anticonceptivos ou em casos de coito desprotegido, deste modo para total eficácia desse medicamento ele deve ser administrado logo após o coito sexual ou em até I2o horas após o ato sexual (LACERDA, et al., 2019).

A CE, dentre os métodos contraceptivos, representa um importante meio de prevenir gravidez indesejada em situações como, falhas de concepção de rotina, violência sexual e até 
mesmo em relações sexuais desprotegidas (OLIVEIRA, et al., 2017). Porém, possui suas desvantagens em relação a seus efeitos colaterais que requer cada vez mais atenção, uma vez que o produto possui fácil acesso, podendo ter seu consumo de forma descontrolada (BRAGA, et al., 2016).

Vieira (2018) relata que o CE é um fármaco que nem sempre é disponibilizado pelo Sistema Único de Saúde (SUS) para usuários da rede pública, levando aquisição direta do medicamento em estabelecimentos de serviços de saúde farmácias ou drogarias particulares sem nenhum tipo de prescrição medica e orientação farmacêutica. Esse acesso facilitado e sem a orientação devida, causa certa preocupação, uma vez que se tem essa substituição pela contracepção de emergência ao invés método contraceptivo mais regular mensal, ocasionando seu uso repetitivo (SANTOS, et al., 2020).

Entretanto o contraceptivo de emergência é seguro e eficaz para a prevenção de gravidez indesejada e torna-se mais eficaz, se sua posologia for seguida corretamente, o CE causa efeitos adversos indesejados que estão relatados na sua bula (VIEIRA, 2018). Embora esse método seja prático, com o uso frequente pode levar a perda ou diminuição da eficácia, desencadeando problemas hormonais, e de infertilidade (VARGAS, et al., 2017).

A maioria das usuárias de CE são adolescentes e jovens que estão em fase de mudanças referente à sexualidade, afinidade e intimidade, porém, é nessa etapa da vida que a maioria das jovens, está em fase de estudos acadêmicos, gerando toda uma expectativa para adentrar no mercado de trabalho, em vista disso, uma gestação não desejada, nesta fase de construção, pode gerar consequências posteriores (COSTA et al., 2020).

Diante disso, este estudo propõe analisar e descrever os possíveis riscos associados ao uso de anticoncepcional de emergência, descrever seus efeitos adversos mais comuns, assim como seu mecanismo de ação, através de uma revisão de literatura.

\section{RESULTADOS E DISCURSÃO}

Assim, dos 31 artigos científicos selecionados nos referidos bancos de dados obtemos os principais resultados, contidos no quadro a seguir. 


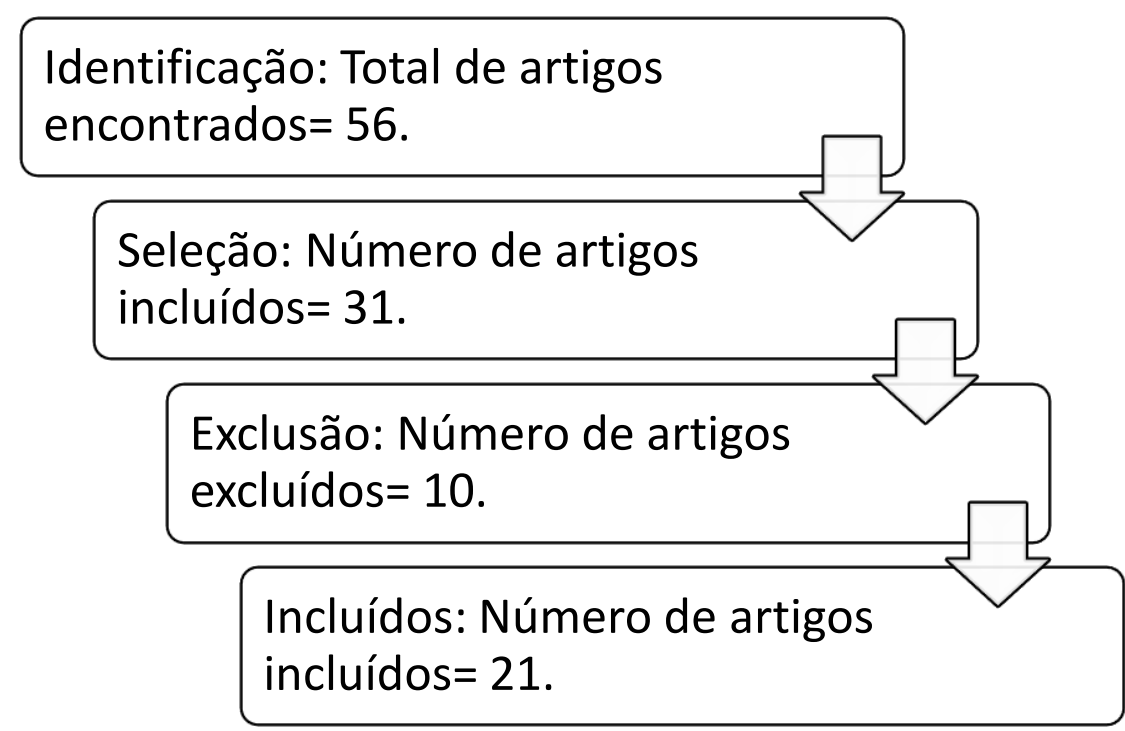

Estudos mostram, que quando se inicia a vida sexual a imaturidade pode levar a uma gravidez indesejada precoce, ou até mesmo outras situações que podem comprometer o futuro dos jovens (BRAGA, et al., 2016). Em relacionamentos estáveis, os jovens tendem a ter mais confiança mútua, portando a preocupação se direciona mais em prevenir uma gravidez, somando na taxa de uso de pílula anticoncepcional (VARGAS, et al., 2017).

O trabalho de Almeida, et al. (2015), relata que cabe ao estado executar ações de saúde, através do SUS (Sistema Único de Saúde) fornecendo e orientando o uso dos métodos contraceptivos, como, a pílula anticoncepcional oral, a mini-pílula, o injetável hormonal, o DIU, o diafragma e o preservativo masculino.

Já Lacerda, et al. (2018), descreve que, no Brasil, em I2 de janeiro de 1996 foi sancionado o Planejamento Familiar (PF), sob a Lei n. 9.263, que contribui para uma educação sexual segura e saúde reprodutiva de qualidade, através de programas educativos e distribuição de métodos contraceptivos, tendo a mulher como protagonista, pois em concordância com os profissionais de saúde, pode escolher o melhor método.

Teve-se então um aumento no uso de métodos contraceptivos, junto com a Contracepção de Emergência (CE), através do acesso facilitado nas farmácias e drogarias, sem prescrição médica, no ano de 2000, o Ministério da Saúde ofertou a CE para atender mulheres vítimas de violência sexual, que logo passou a fazer parte do PF (CARMO, et al., 2017). 
Segundo Almeida, et al (2015), estudantes universitárias tem uma visão diferente sobre Planejamento Familiar, para elas a formação profissional está em primeiro plano, desta forma, estudos mostram que jovens que engravidam durante a vida acadêmica não é planejada e/ou desejada. Entra então a Contracepção Oral de Emergência (COE), como uma solução para jovens adultos evitarem uma gravidez indesejada após práticas sexuais desprotegidas (MONTEIRO, et al., 2019).

O CE, ou pílula do dia seguinte, como é conhecido, surgiu dos estudos de Albert Yuzpe, entre 1960 e 1970, como resposta médica para prevenir gravidez decorrente de estupros (COSTA, et al., 2020). Esse método consiste na ingestão de uma substancia hormonal, o levonorgestrel isolado, disponível em uma ou duas doses, e deve ser administrado após a relação sexual (CARMO, et al., 2017).

Um dos motivos de dificultam seu efeito é o intervalo de tempo entre a relação sexual e seu consumo, que não deve exceder $72 \mathrm{~h}$, quanto mais rápido for administrado maior será sua eficácia (ALMEIDA, et al., 2015). Podendo ser utilizado em qualquer fase do ciclo menstrual, atuando de duas formas como mecanismo de ação, são elas: a primeira retarda ou impede a ovulação, e a segunda modifica a viscosidade do muco cervical, dificultando a locomoção do espermatozoide, impedindo a fertilização (LACERDA, et al., 2019).

O autor Vieira (2018), informa que, segundo as orientações de segurança da saúde, existem anticoncepcionais de uso contínuos seguros e eficazes, sendo que o CE é um medicamento com finalidade apenas para uso emergencial o mesmo não deve ser utilizado com frequência, deve-se respeitar o intervalo de tempo entre uma administração e outro.

No Brasil a Agência Nacional de Vigilância Sanitária (ANVISA), consta no Manual de Assistência ao Planejamento Familiar do MS de 1996, e na Política Nacional de Saúde da Mulher do Ministério da Saúde, que o CE é aprovado e engloba as normas técnicas para assistência às mulheres vítimas de violência sexual (RIECHEL, ET AL, 2016).

Essa metodologia contraceptiva é recomendada somente em casos emergências especiais, quando ocorrer o uso inadequado do anticoncepcional regular como o esquecimento de dois ou mais comprimidos; falha no anticoncepcional, ejaculação após rompimento da camisinha, diafragma se deslocar, relação sexual desprotegida, coerção 
sexual, bem como no cálculo incorreto do período fértil, atraso da aplicação do anticoncepcional mensal. (SANTOS, et al., 2020).

Entretanto, o mecanismo de ação do CE ainda não é preciso, mas sabe-se que ele altera processos fisiológicos da fecundação. Previne a ocorrência da ovulação, interfere na fertilização, com o transporte do embrião para o útero, ou inibe a implantação no endométrio. (POLI, et al., 2009).

O autor Cordeiro (2005), também relata que o mecanismo de ação não se encontra totalmente elucidado, porém o fármaco propõe que a sua ação depende da altura do ciclo em que a mulher o tomar, se o CE for administrado na fase pré-ovulatória evita ou atrasa a ovulação impedindo a fecundação, se administrado pós ovulação e concepção, fara com que o ser gerado não tenha condições de se implantar no útero materno impedindo a continuação da gravidez.

Vieira, (2018), relata que cento e vinte horas após a concepção inicia-se a geração de um embrião e alerta que o uso do CE pode causar alteração no ciclo menstrual, atrasar ao adiantar a ovulação e até mesmo causar anormalidade no organismo reprodutor.

Em concordância, Poli, et al., (2009), destaca que, o CE na composição levonorgestrel (LNG) tem alta eficácia, quando sua posologia prescrita for em duas doses de setecentos e cinquenta $\mu \mathrm{g}$, com intervalo de doze horas ou em dose única de mil e quinhentos $\mu \mathrm{g}$, em ambos a dose deve ser administrada pós coito antecedendo as setenta e duas horas, estudos relatam que quanto antes tomar o CE maior a eficácia e ainda dispõe de vantagens diminuído ao anulando as possíveis reações adversas ocasionadas pelo CE. O estudo de Hafi, et al., (2020), relata o intervalo de tempo seguro para ingestão de uma dose e outra, deve ser administrada mínimo em trinta dias, no período de tempo entre as doses se possível estender ao máximo possível esse prazo.

De acordo com a OMS, o CE é um método de alta eficácia, pois, seus índices de erros são de $2 \%$ para administração em até 24 horas pós-coito, e 4,I\% para administração no intervalo de 25 a 48 horas pós-coito e 4,7\% dentro de 49 e 72 horas, deste modo sua eficácia é variável, pois o tempo de administração da pílula interfere diretamente no resultado, ainda existem formas mais seguras para evitar uma gravidez indesejada, estima-se que no Brasil 
22,1\% das mulheres utilizam anticoncepcional oral, 12,9 preservativo masculino (camisinha), $3,5 \%$ dispositivo intrauterino (DIU), e cerca de $2,4 \%$ tabelinhas e coito interrompido (SOUSA E CIPRIANO, 2019).

A Organização Mundial de Saúde (OMS) relata que o CE tem alta concentração hormonal, seu uso em demasia pode prejudicar a fertilização do óvulo, em vista disso ele é contra indicado para mulheres que desejam engravidar futuramente e para grávidas que possuem riscos de doenças ginecológicas causadas por diferentes parceiros, paciente com risco de trombose vascular, tromboembolismo, diabéticos com complicação vascular, hipertensão descontrolada, doenças sanguíneas, pessoas obesas, mulheres que tem o fluxo menstrual em excesso (VIEIRA, 2018).

Em concordância com o trabalho de Figueredo, et al (2007), o CE é uma verdadeira bomba de hormônios é pode conter dez vez mais hormônios comparados ao contraceptivo convencional, o uso abusivo pode causar vários danos ao organismo feminino, bem como, efeitos adversos que envolvem além de náuseas e vômitos, efeitos tromboembólicos e outras reações tensão mamarias, hemorragia vaginal, fadiga, cefaleias, vertigens, astenia e dores na pélvica.

De acordo com estudos realizados por Alano, et al, (2012), as reações adversas estão presentes de forma considerável com alterações significantes, porém momentâneas, se destacando durante o ciclo menstrual o que é comum após o uso CE, juntamente com náuseas, em sua pesquisa foram coletados os seguintes dados: participaram da pesquisa 360 mulheres com idade média entre 18 e 45 anos, destas $48,6 \%$ já fizeram uso do método aos quais $48,8 \%$ apresentaram alteração no ciclo menstrual e náuseas, no próprio estudo teve um caso em que a entrevistada relatou ter feito o uso do CE várias vezes (não especificou a quantidade), causando a ineficácia do medicamento resultando em uma gravidez, além de efeitos como: náuseas, vômitos, sangramentos, cólicas, cefaleia e alterações no ciclo menstrual.

O uso constante do CE, não associado ao uso de preservativos podem desencadear as Doenças Sexualmente Transmissíveis (DSTs) tais como AIDS, gonorreia e sífilis (SANTOS, et al, 2020). Em concordância com Porto, et al, (2019), esse método de prevenção 
a gravidez em casos de coito desprotegido ocasiona vulnerabilidade aos seus usuários, pois os mesmos ficam expostos as DSTs.

\section{CONCLUSÃO}

O trabalho apresentado relata que o CE é uma substancia hormonal a base de levonorgestrel, considerado como uma bomba de hormônios, esse método tem como função impedir uma possível gravidez indesejada após coito desprotegido. Contudo deve ser usado com cautela, já que o mesmo provoca uma sobrecarga hormonal no organismo feminino, gerando serias consequências futuras como casos de infertilidade. Os efeitos adversos mais comuns causados pelo CE são: náuseas e vômitos, envolvem também efeitos tromboembólicos e outras reações tensão mamarias, hemorragia vaginal, fadiga, cefaleias, vertigens, astenia e dores na pélvica, isso quando usado em excesso e sem conhecimento prévio, por ser um medicamento de fácil acesso.

\section{REFERÊNCIAS BIBLIOGRÁFICAS}

AL HAFI, Inaam; DA SILVA PENTEADO, Camila Valéria; CHEN, Monica. Riscos associados ao uso consecutivo de método contraceptivo de emergência e mapeamento do consumo em Foz do Iguaçu-PR. Brazilian Journal of Health Review, v. 3, n. 6, p. I8864I8877, 2020 .

ALANO, Graziela Modolon et al. Conhecimento, consumo e acesso à contracepção de emergência entre mulheres universitárias no sul do Estado de Santa Catarina. Ciência \& Saúde Coletiva, v. 17, p. 2397-2404, 2012.

ALMEIDA, Francileuda Batista et al. Avaliação do Uso de Anticoncepcionais de Emergência entre Estudantes Universitários. Revista Brasileira de Educação e Saúde, v. 5, n. 3, p. 49-55, 2015.

BRAGA, Ana Paula da Costa. Efeitos do uso da contracepção de emergência: revisão de literatura. 2016.

BRASIL. M.S. Conselho Nacional de Saúde. Diretrizes e Normas Regulamentada de Pesquisa Social. Comissão Nacional e Ética e Pesquisa CONEP Resolução 196/96 sobre a pesquisa envolvendo seres humanos Brasília, 1996.

CARMO, Márcia Simone Almeida Galindo; DUARTE, Stênio Fernando Pimentel. Perfil das Usuárias de Anticoncepcionais de Emergência: Uma Revisão Sistemática. ID on line REVISTA DE PSICOLOGIA, v. II, n. 35, p. 317-324, 2017. 
CASTEL-BRANCO, Margarida; FIGUEIREDO, Isabel Vitória. Ainda sobre a "pílula do dia seguinte". Mundo farmacêutico, n. 29, p. 40-42, 2007.

CASTEL-BRANCO, Maria Margarida; FIGUEIREDO, Isabel Vitória. Contracepção de emergência-sim ou não?(parte 2). 2005.

COSTA, Rodrigo Junior Farias et al. O uso de contraceptivos de emergência em estudantes de uma instituição de ensino superior de Belém, Pará. Revista Brasileira de Educação e Saúde, v. Io, n. 4, p. 124-130, 2020.

CUNHA, ADRIANA; BORTOLOZZI, FLÁVIO. Uso indiscriminado de contraceptivo de emergência por universitárias no norte do paraná.

LACERDA, Jaciane Oliveira da Silva; PORTELA, Fernanda Santos; MARQUES, Matheus Santos. O Uso Indiscriminado da Anticoncepção de Emergência: Uma Revisão Sistemática da Literatura. ID on line Revista de Psicologia, v. 13, n. 43, p. 379-386, 2019.

MONTEIRO, Ana Margarida et al. Contracepção oral de emergência em estudantes do ensino superior de uma escola de saúde. Adolescência: Revista Júnior de Investigação, v. 6, n. I, p. 18-33, 2019.

OLIVEIRA, Jose Leandro; STARLING, Patrícia Soares; FRANCO, Adriane Jane. avaliação do conhecimento e uso da contracepção de emergência por acadêmicos da área de saúde. ANAIS SIMPAC, v. 9, n. I, 2018.

POLI, Marcelino Espírito Hofmeister et al. Manual de anticoncepção da FEBRASGO. Femina, v. 37, n. 9, p. 459-92, 2009.

PORTO, Marta Soares, et al. Conhecimento e utilização de anticoncepção de emergência por jovens no Brasil: revisão integrativa da literatura. Revista Eletrônica de Farmácia, v. I6, n. E, 2019.

RIECHEL, Tatiana; BRAMBILlA, Andreia; AMADEI, Janete Lane. Contracepção de emergência e universitárias da área da saúde. Revista Sustinere, v. 4, n. 2, p. 253-264, 2016.

SANTOS, Alex Henrique Batista et al. O USO INDISCRIMINADO DO CONTRACEPTIVO DE EMERGÊNCIA: UMA REVISÃO, 2020.

SILVA, Emília Vitória et al. Conhecimento e utilização de anticoncepção de emergência por jovens no Brasil: revisão integrativa da literatura. Revista Eletrônica de Farmácia, v. ı6, n. E, 2019.

SOUSA, Luzilene Gomes; CIPRIANO, Vivian Taís Fernandes. Contraceptivo oral de emergência: indicações, uso e reações adversas. Revista Eletrônica Acervo Saúde, n. 22, p. e665-e665, 2019. 
VARGAS, A. C. et al. Uso indiscriminado de contraceptivo de emergência por universitárias no Norte do Paraná. Brazilian Journal of Surgery and Clinical Research, v. 20, n. I, p. 65-71, 2017.

VIEIRA, Gilson Valente et al. Fatores associados ao uso abusivo do contraceptivo de emergência e seus efeitos indesejados em acadêmicas da área da saúde de uma faculdade de Ariquemes- Rondônia. 2018. 